

\title{
SUDEP and mortality in epilepsy: The role of routinely collected healthcare data, registries, and health inequalities
}

\author{
William Owen Pickrell a, $*$, Michael Patrick Kerr b,**
}

\section{a Swansea University Medical School, Swansea University, Swansea SA2 8PP, United Kingdom of Great Britain and Northern Ireland}

\author{
b Institute of Psychological Medicine and Clinical Neurosciences, Cardiff University School of \\ Medicine, Cardiff, United Kingdom of Great Britain and Northern Ireland \\ abstract
}

Routinely collected data are a powerful research resource and offer the opportunity to further our understanding of epilepsy mortality and Sudden Unexpected Death in Epilepsy (SUDEP). The advantages of using routinely collected data include that it often covers whole populations, is already collected, and can be easily linked to other data sources. A significant disadvantage is the difficulty in obtaining accurate causes of death and correctly identifying cases of SUDEP. Using and linking data from epilepsy death registries can improve the quality of mortality data for research. Epilepsy prevalence, incidence, and mortality rates are associated with socioeconomic deprivation. Further research into understanding the link between deprivation and epilepsy mortality could lead to ways to reduce epilepsy mortality.

\section{Introduction}

One of the many challenges faced in epilepsy mortality research is the accurate collection of information on the frequency and nature of deaths in order to understand causation and factors influencing mortality. Such data can be used in the surveillance of mortality and in other research themes such as in links to issues such as health inequalities. An excellent example of the value of surveillance has come from the various registers of pregnancy in epilepsy which have highlighted the risk from sodium valproate to the unborn child and led to major changes in clinical practice [1].

In this article, we discuss the value of routinely collected data and registries, focusing on their value in research and specifically into the link between mortality and deprivation.

\section{Routinely collected data}

Vast amounts of routinely collected electronic health, social, and personal data are generated throughout the world. These data can be anonymized, linked electronically, and used as a powerful healthcare research tool (data linkage). The ability to analyze routinely collected data depends critically on a secure anonymization process, given that specific individual consent for research has not normally been obtained. Several technological and procedural systems have been established to ensure that linked data can be securely anonymized [2]. An example, which is used at the Secure Anonymised Information Linkage databank (SAIL) at Swansea University is the split file approach, where patient identifiable information is separated from the remainder of the data and separately encrypted [3].

There are several advantages of using routinely collected healthcare data for research when compared to other methods. The data have al-ready been collected and do not depend on recruiting individuals to specific trials, which can be costly and can introduce recruitment bias. Routinely 
collected data are also relatively easily available on a population level. Retrospective cohort studies can be conducted with the above advantages which are a potentially powerful tool to study mortality in epilepsy and SUDEP.

\subsection{Issues when using routinely collected data for epilepsy mortality research}

Using routinely collected data to study epilepsy mortality and SUDEP relies on accurately identifying people with epilepsy. Hospital epilepsy registries or clinical neurology databases often contain reliable epilepsy diagnosis from specialists. However, these registries do not often cover the complete relevant epilepsy population and cannot always easily be used in conjunction with other data. Primary care and hospital epilepsy diagnosis codes are therefore used more frequently to identify people with epilepsy. There are concerns that primary care and hospital epilepsy diagnoses are not always accurately routinely coded, e.g., acute symptomatic seizures, convulsive syncope, or dissociative seizures being coded as epilepsy.

Coding systems do vary significantly from country to country, and sometimes, there are significant differences in healthcare systems within individual countries which are important to consider when using disease codes for research. Several factors can influence the accuracy of epilepsy diagnosis codes such as who enters the codes (clinicians versus administrators) or incentives to maintain accurate disease statistics [4]. Generally though, disease diagnosis and management codes seem to be reasonably reliable in ascertaining people with epilepsy in routinely collected data, especially when combined with other epilepsy information such as regular prescription of anti-epileptic drugs (AEDs) or electroencephalographic (EEG) results (where available). For example, Australian, Italian, American, and UK studies achieved sensitivities of $82-94 \%$ and specificities of $94-100 \%$ when identifying people with epilepsy from routinely collected data [5-8].

The vast majority of deaths will be captured by legal frameworks involving death certificates or equivalent systems which are generally accessible for most routinely collected research systems. They will accurately capture deaths but there are justified concerns about the accuracy of recorded causes of deaths. For example, in a previous study, $22 \%$ of death certificates in England and Wales had an inaccurate cause of death when reviewed by medical examiners [9].

There are even more concerns about the accurate recording of SUDEP on death certification. An expert chart review found that 5.3\%of 399 American out of hospital sudden deaths were SUDEP but seizures or complications of seizures were only recorded as the primary cause of deathinaround $20 \%$ of these cases [10]. A retrospective evaluation of forensic autopsy cases in Maryland, USA found 74 cases of SUDEP but SUDEP was listed as the primary cause of death in only 8 cases [11].A UK national audit of epilepsy-related death found that the cause of death was considered to be inadequately stated in $41 \%$ cases of post-mortem reports [12].

Another issue to consider when analyzing death certificate data is the limitation of some coding systems used in death certification. The International Statistical Classification of Diseases and Related Health Problems (ICD) is a commonly used system for death certification. SUDEP has only been incorporated in the very latest version (ICD-11) released in 2018; all other versions, which are still commonly used, do not contain a code for SUDEP $[13,14]$.

\section{Registries of epilepsy deaths}

Disease-based registries can play a vital role in surveillance in medicine, e.g., the UK epilepsy and pregnancy registry [15], and MBRRACE-UK: Mothers and Babies: Reducing Risk through Audits and Confidential Enquiries across the UK [16]. Epilepsy death registries can augment population level 
data with specific mortality data and bridge the gap caused by the limitations in the accuracy of recording epilepsy deaths as described above. Epilepsy death registries offer: opportunities to accurately capture epilepsy-related deaths, a rich set of valuable data and information from relatives and clinicians, and support to the bereaved contributing sensitive information. To the best of our knowledge, there are five epilepsy death registers; these are summarized in Table 1 and described below.

The Epilepsy Deaths Register (EDR) is owned and managed by the UK-based charity SUDEP Action and is described in detail in the linked article in this issue by Osland and Thomas [17]. Individuals from all over the world can register epilepsy deaths in the EDR, but most of its 750 entries are currently from the UK and Ireland. Bereaved relatives complete information (with appropriate support) relating to the circumstances of the death which can be linked to death certificates.

The EDR for Ireland, setup with a partnership between the charities Epilepsy Ireland and SUDEP Action, allows friends or relatives to register epilepsy deaths [18]. This register is based on the UK EDR.

The North American SUDEP Registry (NASR) was launched in 2011 [19,20]. Family members of people with epilepsy who have had an un-expected death can register their relatives' death with NASR. Family members can be referred to NASR from a variety of sources including clinicians, coroners, or advocacy groups [21]. They then complete a phone interview and are asked to allow NASR permission to view medical records. The North American SUDEP Registry collects tissue samples when appropriate and also enrolls living first degree relatives and control decedents.

Verducci et al. recently analyzed NASR data, highlighting the utility of SUDEP registries [21]. As of June2018, there were530NASR participants with 237 definite and probable cases of SUDEP [21].Of these 237 cases, $40 \%$ had generalized epilepsy and $60 \%$ had focal epilepsy; $11 \%$ were not prescribed antiepileptic medications (AEDs) and $37 \%$ of those prescribed AEDs had reportedly missed their last medication dose. As similar studies have shown, there was a low rate of SUDEP awareness with only $16 \%$ of next of kin being aware of SUDEP. Although the data highlighted clinical features that are known to be associated with SUDEP risk (e.g., young age and frequent generalized tonic-clonic seizures [GTCS]), there were a minority of cases with relatively benign epilepsies ( 3 cases with childhood epilepsy with centrotemporal spikes and 7 cases who had never had a GTCS before SUDEP) [21].

The French network, Réseau Sentinelle Mortalité Epilepsie (RSME), was established in 2010. The network includes approximately 120 practitioners including neurologists, epileptologists, and pediatric neurologists from each region of France. The RSME also includes a national network of bereaved families. Epilepsy deaths are reported by physicians or bereaved families [22]. From January 2010 to October 2018, 275 deaths were recorded. The causes of death were as follows: $78 \%$ SUDEP ( $N=207$ ), $6 \%$ accidental trauma, $5 \%$ drowning, $5 \%$ status epilepticus, $2 \%$ suicide, and $6 \%$ other causes.

The Canadian Pediatric SUDEP Registry (CPSR) was developed through collaboration with the Canadian Pediatric Epilepsy Network and the Canadian Pediatric Surveillance Program [23]. Monthly requests are sent to Canadian pediatricians to report pediatric SUDEP cases. After obtaining consent, data are collected in a similar format to the NASR to enable data sharing.

\section{Table 1}

A summary of the main epilepsy death registers. 


\begin{tabular}{|c|c|c|c|}
\hline Name & Country & Notes & Reference \\
\hline Epilepsy Deaths Register (EDR) & UK & $\begin{array}{l}\text { Deaths can be registered from all over the world. Currently around } 750 \text { entries (including } \\
\text { the Epilepsy Deaths Register for Ireland) }\end{array}$ & Osland and Thomas [17] \\
\hline $\begin{array}{l}\text { The Epilepsy Deaths Register for } \\
\text { Ireland }\end{array}$ & Ireland & $\begin{array}{l}\text { Allows friends or relatives to register epilepsy deaths as part of the EDR } 75 \text { entries (as of } \\
\text { March 2019) }\end{array}$ & https://epilepsydeathsregister. \\
\hline $\begin{array}{l}\text { The North American SUDEP } \\
\text { Registry (NASR) }\end{array}$ & $\frac{\text { North }}{\text { America }}$ & 530 entries in 2018 & $\begin{array}{l}\text { hitp://sudep registry.org [20]; } \\
\text { Verducci et al. [21] }\end{array}$ \\
\hline $\begin{array}{l}\text { Réseau Sentinelle Mortalitế } \\
\text { Epilepsie (RSME) }\end{array}$ & France & 275 entries in 2018 & Donner et al. [22] \\
\hline The Canadian Pediatric SUDEP & Canada & Data collected in a similar format to NASR to allow for ease of data sharing. & Donner et al, [23] \\
\hline
\end{tabular}

\section{Examples of studies using population data}

Fazel et al. used routinely collected Swedish data to retrospectively study premature mortality in people with epilepsy and the role of psychiatric comorbidity [24]. The adjusted odds ratio of premature mortal-ity in the 69,995 people with epilepsy was 11.1 when compared with general population controls and 11.4 when compared with unaffected siblings. Fifteen percent of the deaths were from external causes with $75 \%$ of these individuals having a psychiatric comorbidity.

One approach to account for the inaccurate coding of SUDEP is to re-view the medical notes of possible cases of SUDEP identified in routinely collected data. This is not always possible because of the ethical implications of reidentifying individuals from anonymized routinely collected data but is possible in some healthcare systems.

For example, Sveinsson et al. identified 1890 deaths in 57,775 people with epilepsy in Sweden in 2008 using the national patient register and linked death certificates [25]. All death certificates were screened to identify possible cases of SUDEP, and then a detailed review of medical records was performed to identify definite, probable, and possible cases of SUDEP. They found an incidence of $1.20 / 1000$ patient years of definite or probable SUDEP. They also found that epilepsy was mentioned on the death certificate in only $63 \%$ of cases.

The same authors used a similar method to analyze SUDEP risk in a population cohort over time. They found that the incidence of SUDEP in their population-based cohort decreased by around $7 \%$ per year in a six year follow-up period [26].

An American group demonstrated how registry data can augment population level data to identify cases of SUDEP. Ryvlin et al. studied SUDEP rates in a cohort of 40,443 patients who had vagus nerve stimulator (VNS) implants in the USA between 1988 and 2012 [27]. They used VNS manufacturer registry data about deaths in the cohort together with national cause of death data and an expert panel review process to identify SUDEP cases. There were 632 cases of SUDEP, and similarly to the Swedish study [26], they found that the rate of SUDEP decreased with follow-up time from $2.47 / 1000$ patient years in the first two years after VNS implantation to $1.68 / 1000$ patient years three to ten years after VNS implantation. Because of the limitations of this study, the cause of this finding is not known and could be caused by several factors including the natural long-term dynamic of SUDEP rate, attrition, and the impact of VNS therapy [27].

Where it is not possible to identify or reidentify individuals from population data in order to accurately confirm causes of death or SUDEP, then using epilepsy death registries with accurate causes of death might offer an alternative. Registries containing lists of definite cases of SUDEP or other epilepsy deaths can be linked (or anonymously linked if necessary) to population data; for example, using a split file approach as used in the SAIL databank [3].

\section{Deprivation}


Deprivation can be defined as the lack of opportunities and re-sources which we might expect in society [28]. Deprivation is most accurately measured on an individual basis, e.g., individual income, housing, and access to resources. When this is not possible, then area-based measures of deprivation can be used. Measures of deprivation used in the UK include the Index of Multiple Deprivation (England) and the Welsh Index of Multiple Deprivation (Wales) [28,29]. These in-dices of deprivation calculate a deprivation score for a small geographical area (about 1500 people) based on weighted scores from eight domains. These domains represent different types of deprivation including income (unemployment benefit claimant rate), geographical access to services (proximity to primary care and schools), and health (death rate, cancer rates).

There is an established link between socioeconomic deprivation and epilepsy. Both epilepsy prevalence and incidence are positively correlated with increasing deprivation [30-32](Fig. 1). This suggests that factors associated with deprivation may cause epilepsy (social causation) as opposed to epilepsy itself causing deprivation (social drift) [33] .

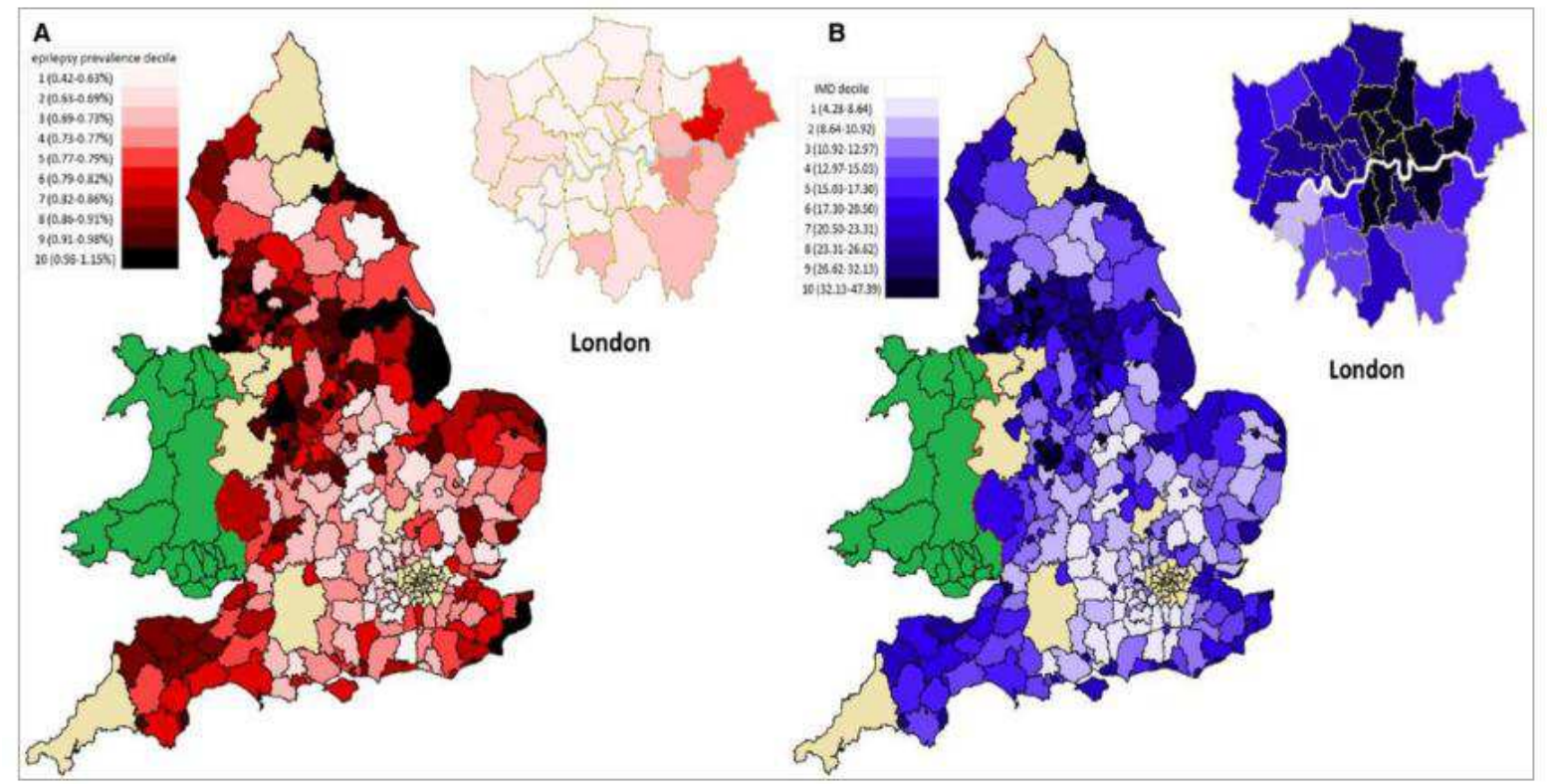

Fig 1. Maps to illustrate ( $\mathrm{A}$ ) epilepsy prevalence (decle rank darker color red = increased prevalence) and (B) variability in deprivation (IMD decile rank darker color blue = increased deprivation) in England, UK. The area shown top right is the City of London in detail (For interpretation of the references to color in this figure legend, the reader is referred to the web version of this article.

Reproduced with permission from [32] (license number: 4597680973500 )

A recent report by Public Health England, covered in more detail elsewhere in this special edition, found that epilepsy-associated deaths in the most deprived areas are nearly three times more likely than in the least deprived areas [34,35]. This association was not found with other major neurological conditions but may be due to the fact that epilepsy prevalence is increased in areas of increased deprivation - if there are more people with epilepsy then there will be more deaths associated with epilepsy. Another possibility is that factors associated with deprivation cause increased mortality. For example, access to healthcare ser-vices is one measure used in indices of multiple deprivation $[28,29]$. Poorer access to healthcare services may mean that more deprived people with epilepsy do not have access to the same support that they re-quire for their epilepsy which may impact on mortality and SUDEP rates.

Future research is needed to try and understand the reasons for this link between deprivation, epilepsy, and mortality. It may be that potentially reversible factors associated with deprivation are associated with epilepsy mortality as well offering the real opportunity to save lives. 


\section{Conclusion}

The use of routinely collected data has the potential to provide a powerful tool to explore population-based influences on epilepsy mortality. This approach will complement the increasingly rich data from registries. Future projects linking registry-identified data with routine datasets promise to improve the quality of routinely collected epilepsy mortality data.

\section{Declaration of Competing interests}

Prof Kerr is vice chair of SUDEP Action. The authors confirm that they have no other conflicts of interest.

\section{References}

[1] Campbell E, Kennedy F, Russell A, Smithson WH, Parsons L, Morrison PJ, et al. Mal-formation risks of antiepileptic drug monotherapies in pregnancy: updated results from the UK and Ireland Epilepsy and Pregnancy Registers. J Neurol Neurosurg Psy-chiatry 2014;85:1029-34.

[2] Jones KH, Ford DV, Jones C, Dsilva R, Thompson S, Brooks CJ, et al. A case study of the Secure Anonymous Information Linkage (SAIL) gateway: a privacy protecting re-mote access system for health-related research and evaluation. J Biomed Inform 2014;50:196-204.

[3] Ford D, Jones K, Verplancke J, Lyons R, John G, Brown G, et al. The SAIL databank: building a national architecture for e-health research and evaluation. BMC Health Serv Res 2009. https://doi.org/10.1186/1472-6963-9-157.

[4] National Institute for Health and Care Excellence (NICE). Quality and outcomes framework indicator. https://www.nice.org.uk/Standards-and-indicators/QOFIndicators;2017.

[5] Tan M, Wilson I, Braganza V, Ignatiadis S, Boston R, Sundararajan V, et al. Develop-ment and validation of an epidemiologic case definition of epilepsy for use with rou-tinely collected Australian health data. Epilepsy Behav 2015;51:6-72.

[6] Franchi C, Giussani G, Messina P, Montesano M, Romi S, Nobili A, et al. Validation of healthcare administrative data for the diagnosis of epilepsy. J Epidemiol Community Health 2013;67:1019-24.

[7] Holden EW, Grossman E, Nguyen HT, Gunter MJ, Grebosky B, Von Worley A, et al. Developing a computer algorithm to identify epilepsy cases in managed care organi-zations. Dis Manag 2005;8:1-14.

[8] Fonferko-Shadrach B, Lacey AS, White CP, Powell HWR, Sawhney IMS, Lyons RA, et al. Validating epilepsy diagnoses in routinely collected data. Seizure 2017;52: 195-8.

[9] Office for National Statistics. Death certification reform: a case study on the potential impact on mortality statistics, England and Wales; 2012.

[10] Chen S, Joodi G, Devinsky O, Sadaf MI, Pursell IW, Simpson Jr RJ. Under-reporting of sudden unexpected death in epilepsy. Epileptic Disord 2018;20:270-8.

[11] Zhuo L, Zhang Y, Zielke HR, Levine B, Zhang X, Chang L, et al. Sudden unexpected death in epilepsy: evaluation of forensic autopsy cases. Forensic Sci Int 2012;223: 171-5.

[12] Hanna NJ, Black M, Sander JWS, Smithson WH, Appleton R, Brown S, et al. National sentinel clinical audit of epilepsy-related death: epilepsy-death in the shadows. The Stationary Office; 2012. 
[13] Jette N, Beghi E, Hesdorffer D, Moshe SL, Zuberi SM, Medina MT, et al. ICD coding for epilepsy: past, present, and future-a report by the International League Against Ep-ilepsy Task Force on ICD codes in epilepsy. Epilepsia. 105;56:348-355.

[14] World Health Organisation. International Classification of Diseases (ICD). https://www.who.int/classifications/icd/en/.

[15] The UK Epilepsy and Pregnancy Register.

http://www.epilepsyandpregnancy.co.uk/home.htm.

[16] MBRRACE-UK: Mothers and Babies: Reducing Risk through Audits and Confidential Enquiries across the UK. https://www.npeu.ox.ac.uk/mbrrace-uk.

[17] Osland K, Thomas RH. Learnings from deaths - the Epilepsy Deaths Register; 2019 [This issue].

[18] The Epilepsy Deaths Register for Ireland. https://epilepsydeathsregister.org/ie.

[19] Louik J, Doumlele K, Hussain F, Crandall L, Buchhalter J, Hesdorffer D, et al. Experi-ences with premorbid SUDEP discussion among participants in the North American SUDEP Registry (NASR). Epilepsy Behav 2017;70:131-4.

[20] The North American SUDEP Registry. http://sudepregistry.org.

[21] Verducci C, Hussain F, Donner E, Moseley BD, Buchhalter J, Hesdorffer D, et al. SUDEP in the North American SUDEP Registry: the full spectrum of epilepsies. Neurology 2019.

https://doi.org/10.1212/WNL.0000000000007778 [Epub ahead of print].

[22] Donner EJ, Waddell B, Osland K, Leach JP, Duncan S, Nashef L, et al. After sudden unexpected death in epilepsy: lessons learned and the road forward. Epilepsia 2016;57 (Suppl. 1):4653.

[23] Donner EJ, Devinsky O. Registries for SUDEP research. In: Panelli R, Hanna J, Jeffs T, Brocett $P$, editors. Continuing the global conversation. SUDEP Action \& SUDEP Aware; 2018 [online]. [retrieved 28/05/2019]. Available from: www. sudepglobalconversation.com.

[24] Fazel S, Wolf A, Långström N, Newton CR, Lichtenstein P. Premature mortality in ep-ilepsy and the role of psychiatric comorbidity: a total population study. Lancet 2013; 382:1646-54.

[25] Sveinsson O, Andersson T, Carlsson S, Tomson T. The incidence of SUDEP: a nation-wide population-based cohort study. Neurology 2017;89:170-7.

[26] Tomson T, Sveinsson O, Carlsson S, Andersson T. Evolution over time of SUDEP inci-dence: a nationwide population-based cohort study. Epilepsia 2018;59:e120-4.

[27] Ryvlin P, So EL, Gordon CM, Hesdorffer DC, Sperling MR, Devinsky O, et al. Long-term surveillance of SUDEP in drug-resistant epilepsy patients treated with VNS therapy. Epilepsia 2018;59:562-72.

[28] Welsh Index of Multiple Deprivation (WIMD). Revised. Statistics for Wales. https://gov.wales/statistics-and-research/welsh-index-multiple-deprivation/?skip= 1\&lang=en;2014.

[29] English indices of deprivation. https://www.gov.uk/government/statistics/english-indices-ofdeprivation-2015; 2015. 
[30] Morgan CL, Ahmed Z, Kerr MP. Social deprivation and prevalence of epilepsy and as-sociated health usage. J Neurol Neurosurg Psychiatry 2000;69:13-7.

[31] Heaney DC, MacDonald BK, Everitt A, Stevenson S, Leonardi GS, Wilkinson P, et al. Socioeconomic variation in incidence of epilepsy: prospective community based study in south east England. BMJ 2002;325:1013-6.

[32] Steer S, Pickrell WO, Kerr MP, Thomas RH. Epilepsy prevalence and socioeconomic deprivation in England. Epilepsia 2014;55:1634-41.

[33] Pickrell WO, Lacey AS, Bodger OG, Demmler JC, Thomas RH, Lyons RA, et al. Epilepsy and deprivation, a data linkage study. Epilepsia 2015;56:585-91.

[34] Paul Morrish, Susan Duncan, Hannah Cock. Article in this edition describing the pub-lic health England report into mortality in neurological conditions.

[35] Public Health England. Deaths associated with neurological conditions; 2018. 